\title{
Multidrug Resistant Salmonella typhi and Antimicrobial Agent Quality and Bioavailability
}

Increasing incidence of multidrug resistant Salmonella typhi has been a scourge for those afflicted with enteric fever all over the world. Recently Gautam et al. [1] reported decreased Salmonella typhi susceptibility to ciprofloxacin. The minimum inhibitory antibiotic concentration for third generation cephalosporin and aminoglycosides was above the cut-off level. Plasmid and DNA genomic characterization of 102 Salmonella enterica serovar Typhi isolates from different regions of Kenya was intriguing. There were only $13.7 \%$ fully susceptible isolates, with $82.4 \%$ being resistant to five commonly available drugs [2]. In all probability, more expensive and not readily available antimicrobials will be required to address future episodes of multi-drug resistant $S$. enterica serovar Typhi strains. For rational use of these, as well as the commonly available antibiotics, it is essential to examine bioavailability and field quality as well as the types of antibiotics offered to patients.

The efficacy of intramuscularly given benzathine penicillin $\mathrm{G}$ and oral penicillin $\mathrm{V}$ for the eradication of streptococci from children with acute pharyngitis has been known for a while. Recently, however, there was a warning from Minneapolis, Minnesota, where the recommended penicillin doses applied to children with group A streptococci infection were accompanied by $35 \%$ to $37 \%$ failures. A carrier state and inadequate bioavailability of intramuscularly given benzathine penicillin were responsible for this poor therapeutic response [3].

Different brands of orally administered antibiotics vary remarkably in their in vivo bioavailability Dissolution efficiencies of different brands of film coated ciprofloxacin tablets were monitored in acetic acid and phosphate buffer $\mathrm{pH} 7$.4. Five of the six brands with a $60-70 \%$ fall in 30 minutes were adequately bio-

Address for correspondence: Dr. Subhash C. Arya. Sant Parmanand Hospital, 18 Alipore Road. Delhi- 110054, India. Email: subhashji@hotmail.com available in vivo. The sixth brand had less than a $40 \%$ fall in ciprofloxacin content and was less bioavailable [4]. Poor bioavailability would result in therapeutic failures and would promote the emergence of multi drug resistant S. enterica serovar Typhi.

Assays made in Nigeria, of the active ingredients of common therapeutic agents, including antibiotics offered to patients with various ailments in pharmacies, gave astounding results. As many as $48 \%$ of the samples of common medicines were found outside the limits prescribed by the British Pharmacopoeia [5]. Similar scenarios with common or not so common antimicrobials offered against S. enterica serovar Typhi episodes would favor the selection, propagation and dissemination of multi drug-resistant isolates. Certainly, surveillance of antimicrobial potency and bioavailability of prescriptions against typhoid would reduce therapeutic failures attributed to poor quality medicines or any inadequate bioavailability.

$$
\begin{aligned}
& \text { Subhash C. Arya } \\
& \text { Nirmala Agarwal }
\end{aligned}
$$

\section{References}

1. Gautam V., Gupta N.K., Chaudhary U., Arora D.R. Sensitivity pattern of salmonella serotypes in Northern India. Braz J Infect Dis 2002 6:281-7.

2. Kariuki S., Revathi G., Muyodi J., et al. Characterization of multidrug-resistant typhoid outbreaks in Kenya. J Clinical Microbiol 2004;42:1477-82.

3. Kaplan E.L., Johnson D.R. Unexplained reduced microbiological efficacy of intramuscular benzathine penicillin $\mathrm{G}$ and of oral penicillin $\mathrm{V}$ in eradication of group A streptococci from children with acute pharyngitis. Pediatrics 108:180-6.

4. Ofoeule S.I., Udeogaranya P.O., Okonata J.M. Prediction of in vivo bioavailability of six brands of ciprofloxacin film coated tablets using the concept of dissolution efficiency (DE). Boll Chim Farm 2001;140(3):187-91.

5. Taylor R.B, Shakoor O., Behrens R.H., et al. Pharmacopoeial quality of drugs supplied by Nigerian pharmacies. Lancet 2001;357:1932-6. 